\title{
ELUCIDACIÓN ESTRUCTURAL DEL ACEITE ESENCIAL DE Ruta Graveolens L. RUDA, ACTIVIDAD ANTIOXIDANTE Y BIOENSAYO DE CITOTOXICIDAD
}

\author{
Structural elucidation of essential oil of Ruta graveolens L. Ruda, antioxidant activity and \\ cytotoxicity bio-assay
}

Américo J Castro L ${ }^{1}$, José R Juárez E ${ }^{1}$, Norma J Ramos $C^{1}$, Silvia Suárez $C^{2}$, Fernando Retuerto $\mathrm{P}^{3}$, Sixto A Gonzales $\mathrm{E}^{4}$ ${ }^{1}$ InstitutodeCiencias Farmacéuticasy Recursos Naturales “Juan de Dios Guevara”- Facultad de Farmaciay Bioquímica. ${ }^{2}$ Centrode Investigación de Bioquímica y Nutrición "Alberto Guzmán Barrón”-Facultad de Medicina. ${ }^{3}$ Instituto de Investigación "Antonio Raimondi” Facultad de

Ciencias Biológicas. Universidad Nacional Mayor de San Marcos. ${ }^{4}$ Instituto de Medicina Legal, Ministerio Público-Fiscalía de la Nación

\section{RESUMEN}

El objetivo del estudio fue realizar la elucidación estructural del aceite esencial de Ruta graveolens L. Ruda, determinar su actividad antioxidante y su citotoxicidad. Las plantas completas se colectaron en la provincia de Tarma, Región Junín, aislándose de ellas $1,80 \mathrm{~kg}$ de hojas frescas que fueron tratadas en un sistema de arrastre con vapor de agua, obteniéndose un rendimiento de $0,3 \% \mathrm{v} / \mathrm{p}$ de aceite esencial. La elucidación estructural de los componentes químicos del aceite esencial se realizó por Cromatografía de Gas/Espectrometría de Masas (CG-EM), destacando la identificación de los siguientes componentes: 2-octanona, 2-nonanona, nonanal, ciclohexen-5,6-dietenil-1-metil, 2-decanona, metil nonyl éter, 2-undecanona, ciclotetradecano, 2-dodecanona y 2-tridecanona. La evaluación de la actividad antioxidante in vitro del aceite esencial se realizó utilizando el método de captación del radical 2,2-difenilpicrilhidrazil (DPPH), con lo cual se demostró su capacidad antioxidante como donador de electrones o hidrógeno al radical DPPH. El IC 50 del aceite esencial, $16,13 \mathrm{mg} / \mathrm{mL}$, es elevado comparado con el ácido ascórbico que es de 2,4 $\mu \mathrm{g} / \mathrm{mL}$ y la captación del DPPH es relativamente baja. La citotoxicidad del aceite esencial sobre el desarrollo embrionario de Tetrapygus niger, erizo de mar negro, se manifestó a concentraciones de 50 y $75 \mu \mathrm{L}$ a través de óvulos alterados, lisis, retraso en el desarrollo y embriones que no clivaron; a la concentración de $100 \mu \mathrm{L}$ se detuvo el desarrollo.

Palabras clave: Ruta graveolens L., Tetrapygus niger, elucidación estructural, actividad antioxidante, citotoxicidad.

\section{SUMMARY}

Study objective was performing the structural elucidation of the essential oil of Ruta graveolens L. Ruda, determine its antioxidant activity and cytotoxicity testing. The complete plants were collected in Tarma, Region of Junin, and provided 1,8o $\mathrm{kg}$ of fresh leaves that were treated in a drag system with water vapor, obtaining performance of o, $3 \% \mathrm{v} / \mathrm{p}$ essential oil perfusion. The structural elucidation of chemical constituents of essential oil was performed by Gas Chromatography - Mass Spectrometry (GC-MS), stressing the identification of the following chemical components: 2-octanona 2-nonanona, nonanal, ciclohexen-5, 6-dietenil-1-methyl-, 2-decanona, nonyl ether, methyl 2-undecanona, ciclotetradecano, 2-dodecanona and 2-tridecanona. Evaluation of antioxidant activity in vitro of essential oil was made with the radical 2, 2-difenilpicrilhidrazil (DPPH) uptake method, demonstrating the radical DPPH antioxidant capacity as donor of electrons or hydrogen. The IC50 of essential oil was $16,13 \mathrm{mg} / \mathrm{mL}$, high compared with acid ascorbic value, $2,4 \mu \mathrm{g} / \mathrm{mL}$, and the capture of DPPH is relatively low. The cytotoxicity of essential oil on the embryonic development of Tetrapygus niger, black sea urchin, showed at concentrations of 50 and $75 \mu \mathrm{L}$ altered eggs, lysis, delayed development and embryos that not made division. To concentration of $100 \mu \mathrm{L}$ stopped development.

Keywords: Ruta graveolens L, Tetrapigus niger, structural elucidation, antioxidant activity, cytotoxicity.

\section{INTRODUCCIÓN}

$\mathrm{R}$ uta graveolens L. Ruda, es una planta nativa originaria del sur de Europa y Asia menor; se le atribuyen efectos medicinales como: antiséptico, estimulante, emenagogo, antiespasmódico, carminativo, diaforético, hipotensory sedante. En la medicina tradicional se utiliza para dolores reumáticos, faringitis, rinitis, amenorrea, menorragia, flatulencia y como abortivo ${ }^{(1)}$.

Los aceites esenciales se encuentran presentes en muchas especies vegetales y se ubican en diferentes partes de la planta. Están químicamente constituidos por monoterpenos, sesquiterpenos y compuestos aromáticos; sonodoríferoseinmisciblesenagua. Seobtienenpordiversas técnicas de extracción como: utilizando sustancias grasas, con solventes orgánicos, por prensado, hidrodestilación, por arrastre con vapor de agua y por fluidos supercríticos.
Tienen aplicación en la industria de alimentos, cosmética y farmacéutica. Las técnicas más usuales para determinar su análisis cuantitativo y cualitativo es por Cromatografía de Gas/Espectrometría de Masas (CG/EM) ) ${ }^{(2,3)}$. Las técnicas más usuales para determinar su análisis cualitativo y cuantitativo es por Cromatografía de Gas/Espectrometría de Masas (CG/EM) 2,3. La actividad antioxidante del aceite esencial de la especie estudiada fue determinada por el método de captación del radical 2,2-difenilpicrilhidrazil (DPPH) y la actividad citotóxica se observó en el desarrollo embrionario de Tetrapygus niger, erizo de mar negro.

\section{MATERIAL Y MÉTODOS}

La especie vegetal se colectó en la provincia de Tarma, región Junín, estableciéndose la siguiente clasificación botánica, según el sistema de clasificación 
de Engler \& Prantl, modificado por Melchior en 1964:

\begin{tabular}{|lll|}
\hline División & $:$ & Angiospermas \\
Clase & $:$ & Dicotiledónea \\
Subclase & $:$ & Arquiclamídeas \\
Orden & $:$ & Rutales \\
Género & $:$ & Ruta \\
Especie & $:$ & Ruta graveolens \\
N.V. & $:$ & Ruda \\
\hline
\end{tabular}

\section{Análisis por Cromatografía de gas/Espectrometría de masa (CG/EM)}

El análisis se realizó en un Cromatógrafo de Gas/ Espectrómetro de Masa (CG-EM) modelo CG: Trace / EM: Trace, provisto de una columna capilar de silicagel de 30 metros de longitud. Las condiciones utilizadas fueron: temperatura inicial $40{ }^{\circ} \mathrm{C}, 10{ }^{\circ} \mathrm{C} / \mathrm{min}$; temperatura final 110 ${ }^{\circ} \mathrm{C}$ (1 min), temperatura del inyector $240{ }^{\circ} \mathrm{C}$ y volumen de inyección $5 \mu \mathrm{L}$. Laidentificación delos componentesquímicos del aceite se realizó mediante comparación computarizada de sus espectros de masa con los de la base de datos del equipo.

\section{Determinación in vitro de la actividad antioxidante del aceite esencial}

Se realizó utilizando el método del radical 2,2-difenilpicrilhidrazil (DPPH), en un espectrofotómetro UV-VIS Double Beam PC. El DPPH es un radical libreestable que presenta un color púrpura con absorbancia a $517 \mathrm{gm}$. Las sustancias atrapadoras de radicales libres (donadoras de hidrógeno) reaccionan con este compuesto y producen la desaparición del color. La reacción se siguió midiendo la disminución de la absorbancia a $517 \mathrm{gm}$. Los resultados se expresaron como $\mathrm{IC}_{5}$ o, porcentaje de inhibición, porcentaje de actividad antirradicalaria o equivalentes a la vitamina $C$. El DPPH se disolvió en metanol a una concentración de 20 $\mu \mathrm{g} / \mathrm{mL}$. La muestra se preparó a concentraciones de 100 , 50 y $10 \mu \mathrm{g} / \mathrm{mL}$ y se empleó como sustancia atrapadora de referencia al ácido ascórbico. Los resultados se expresaron según la siguiente fórmula:

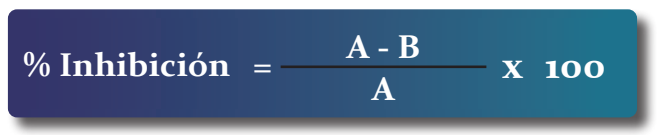

Donde:

$\mathrm{A}=$ lectura del DPPH

$\mathrm{B}=$ lectura del aceite esencial

Se determinó el $\mathrm{IC}_{50}$ para el ácido ascórbico y para el aceite esencial de Ruta graveolens $\mathrm{L}$.

\section{Determinación de la actividad citotóxica \\ Material biológico}

- Ruta graveolens L., Tetrapygus niger, erizo de mar negro El bioensayo se realizó en 22 muestras de erizo de mar negro Tetrapygus niger, las que fueron colectadas en las playas de Ancón, ubicada a $43 \mathrm{~km}$ al norte de Lima, y trasladadas en agua de mar entre 10 y $15^{\circ} \mathrm{C}$.

\section{Procesamiento de las muestras}

Obtención de gametos. Se abrió cuidadosamente al erizo por la parte dorsal, con ayuda de una tijera de disección, cortando en circunferencia, para no dañar los gametos. El sexo del animal se determinó al observar el color de los gametos. Los óvulos de Tetrapygus niger son de color granate y el esperma es cremoso. Se extrajeron los racimos de óvulos de tres erizos hembras con ayuda de una pinza limpia, y se colocaron en una solución de $500 \mathrm{~mL}$ de agua de mar fría hasta que se tiñeran a un color púrpura. Se utilizó un oxigenador para mantener los óvulos viables, después de lo cual se procedió a lavarlos decantando el agua sobrenadante con la finalidad de eliminar el tejido celómico y restos internos que se encontraban adheridos a los mismos, recambiando el agua de mar, previamente filtrada 3 veces, para luego completar la solución a $250 \mathrm{~mL}$.

Fertilización. Se agregó una gota de espermatozoides con ayuda de una pipeta Pasteur, sobre un vaso de precipitados que contenía la suspensión de óvulos lavados listos para ser fecundados. Se mezclaron suavemente los espermatozoides y los óvulos con una pipeta Pasteur limpia. Posteriormente se transfirió una muestra de la suspensión de óvulos y espermatozoides a una lámina portaobjeto. Se colocó un cubreobjetos y se observó al microscopio compuesto a 4oX. La observación delóvulo fecundado significóqueel procedimiento de fertilización se realizó en condiciones óptimas.

Preparación del extracto. Dos $\mathrm{mL}$ del aceite esencial se diluyeron con un $\mathrm{mL}$ de agua de mar fría (solución madre). De la solución madre se prepararon diluciones, de: 10, 25, 50, 75, 100, 150 y $200 \mu \mathrm{L}$ en diez $\mathrm{mL}$.

Preparación de las muestras en viales. Se prepararon diluciones por duplicado y como control se utilizó el aceite esencial y el dimetilsulfóxido (DMSO). Se agregó a cada vial un $\mathrm{mL}$ de agua de mar fría (filtrada de dos a tres veces). Luego se tomaron dos $\mathrm{mL}$ de la suspensión de óvulos recién fecundados y se añadieron a cada vial de la batería. Se agregaron $10 \mu \mathrm{L}$ de DMSO a todos los viales exceptuando al control. Una vez preparada la batería de viales con las

Tabla 1. Composición química del aceite esencial de Ruta graveolens L., determinado por Cromatografía de Gases/Espectrometría de Masas (CG/EM).

\begin{tabular}{lc}
\hline \multicolumn{1}{c}{ Composición química } & $\begin{array}{c}\text { Tiempo de retención (TR) } \\
\text { (minutos) }\end{array}$ \\
\hline 2 - octanona & 1,41 \\
2 - nonanona & 1,89 \\
Nonanal & 1,96 \\
Ciclohexen - 5,6-dietenil - 1 - metil & 2,27 \\
2 - decanona & 2,51 \\
Metil nonyl éter & 2,71 \\
2 - undecanona & 3,34 \\
Ciclotetradecano & 3,39 \\
2 - dodecanona & 3,95 \\
2 - tridecanona & 5,23 \\
\hline
\end{tabular}


Tabla 2. Porcentaje de captación DPPH del aceite esencial.

\begin{tabular}{ccc}
\hline $\begin{array}{c}\text { Concentración } \\
\boldsymbol{\mu L} / \mathbf{m L}\end{array}$ & $\begin{array}{c}\text { Absorbancia } \\
\mathbf{5 1 7} \mathbf{~} \mathbf{~ m}\end{array}$ & $\mathbf{\%}$ \\
\hline 0 & 0,400 & - \\
6 & 0,332 & 17,00 \\
12 & 0,279 & 30,15 \\
18 & 0,240 & 40,00 \\
24 & 0,207 & 48,25 \\
30 & 0,180 & 55,00 \\
\hline
\end{tabular}

Tabla 3. Porcentaje de captación DPPH del ácido ascórbico.

\begin{tabular}{ccc}
\hline $\begin{array}{c}\text { Concentración } \\
\mathbf{m g} / \mathbf{m L}\end{array}$ & $\begin{array}{c}\text { Absorbancia } \\
\mathbf{5 1 7} \mathbf{~ j m}\end{array}$ & $\mathbf{\%}$ \\
\hline 2,4 & 0,265 & 50 \\
2 & 0,316 & 41,26 \\
1,6 & 0,364 & 32,34 \\
1,2 & 0,409 & 23,88 \\
0,8 & 0,459 & 14,59 \\
0 & 0,538 & - \\
\hline
\end{tabular}

Tabla 5. Niveles de dilución del aceite esencial de Ruta graveolens L.

\begin{tabular}{|c|c|c|c|c|c|}
\hline $\begin{array}{c}\text { Número } \\
\text { vial }\end{array}$ & $\begin{array}{c}\text { Agua de } \\
\text { mar }(\mathrm{mL})\end{array}$ & $\begin{array}{c}\text { Suspensión de } \\
\text { huevos (mL) }\end{array}$ & $\begin{array}{l}\text { Dilución aceite } \\
\text { esencial }(\mu \mathrm{L})\end{array}$ & $\begin{array}{l}\text { Volumen } \\
\text { DMSO }(\mu \mathrm{L})\end{array}$ & Citotoxicidad \\
\hline 1 y 7 & 1 & 2 & 200 & 10 & $\mathrm{M}$ \\
\hline 2 y 8 & 1 & 2 & 100 & 10 & M \\
\hline 3 у 9 & 1 & 2 & 50 & 10 & $\mathrm{~V}$ \\
\hline 4 y 10 & 1 & 2 & 25 & 10 & V \\
\hline 5 (control) & 1 & 2 & - & - & V \\
\hline 6 (DMSO) & 1 & 2 & - & 10 & V \\
\hline 11 y 14 & 1 & 2 & 150 & 10 & M \\
\hline 12 y 15 & 1 & 2 & 75 & 10 & M \\
\hline 13 y 16 & 1 & 2 & 10 & 10 & $\mathrm{~V}$ \\
\hline
\end{tabular}

$\mathrm{M}=$ Muertos, $\mathrm{V}=$ Vivos

muestras correspondientes, se acondicionaron en una cámara con agua helada a temperatura entre 10 y $15^{\circ} \mathrm{C}$. Se tomaron dos muestras cada dos horas, hasta observar cuál era la dilución en la que se producía el mayor porcentaje de células muertas durante el desarrollo del embrión de erizo de mar negro.

\section{RESULTADOS}

El método de destilación por arrastre con vapor de agua permitió obtener un rendimiento de $0,3 \% \mathrm{v} / \mathrm{p}$ del aceite esencial de hojas frescas de Ruta graveolens L. Se realizó el análisis preliminar del aceite observándose un líquido ligeramente amarillo, aromático, penetrante y altamente volátil, miscible en alcohol etílico absoluto, en éter etílico y n-hexano, e inmiscible en agua. El análisis de la composición química del aceite esencial permitió identificar 10 componentes químicos que se presentan en la figura 1 y tabla 1 . Los resultados obtenidos en la determinación de la actividad antioxidante se presentan en las tablas 2,3 y 4 y figuras 2 y 3. La determinación de la actividad citotóxica, en los niveles de dilución del aceite esencial en microlitros $(\mu \mathrm{L})$, se realizó por dos veces cada dos horas; presentando los resultados en la tabla 5 .

\section{DISCUSIÓN}

La composición química que presentan los aceites esenciales está constituida especialmente por
Tabla 4. IC50 del aceite esencial de Ruta graveolens $\mathrm{L}$.

\begin{tabular}{cc}
\hline & IC50 \\
\hline Aceite de ruda & $16,13 \mathrm{mg} / \mathrm{mL}$ \\
Ácido Ascórbico & $2,4 \mu \mathrm{g} / \mathrm{mL}$ \\
\hline
\end{tabular}

terpenos, sesquiterpenos y compuestos aromáticos, que les confieren actividad antioxidante. Los componentes químicos elucidados en el aceite esencial de las hojas frescas de Ruta graveolens L., resultan ser en su mayoría alifáticos y de tipo cetónico, muy similares a los obtenidos por cromatografía de gases del el aceite esencial de Ruta graveolens L. de Mérida, Venezuela, que presenta como compuestos mayoritarios 2-undecanona (43,0\%) y 2-nonanona (33,5\%) (4). Asimismo, la composición química determinada resultó ser similar, en sus componentes mayoritarios, a la de Ruta graveolens L. y Ruta chalepensis L. estudiadas en otros países como Italia, Irán y Argelia ${ }^{(5,6)}$. En el Perú, investigaciones realizadas con el extracto acuoso de hojas de Ruta graveolens L. demostraron que presenta actividad mutagénica en el proceso realizado sin activación metabólica con cepas bacterianas ${ }^{(7)}$. De igual manera, de las hojas molidas y tratadas con hidróxido de amonio y diclorometano se ha aislado un alcaloide acridónico identificado como arborinina y dos alcaloides furoquinolénicos, uno de los cuales posee actividad mutagénica y fotomutagénica comprobada ${ }^{(8)}$.

La baja actividad antioxidante del aceite esencial en nuestro estudio, determinada por el método

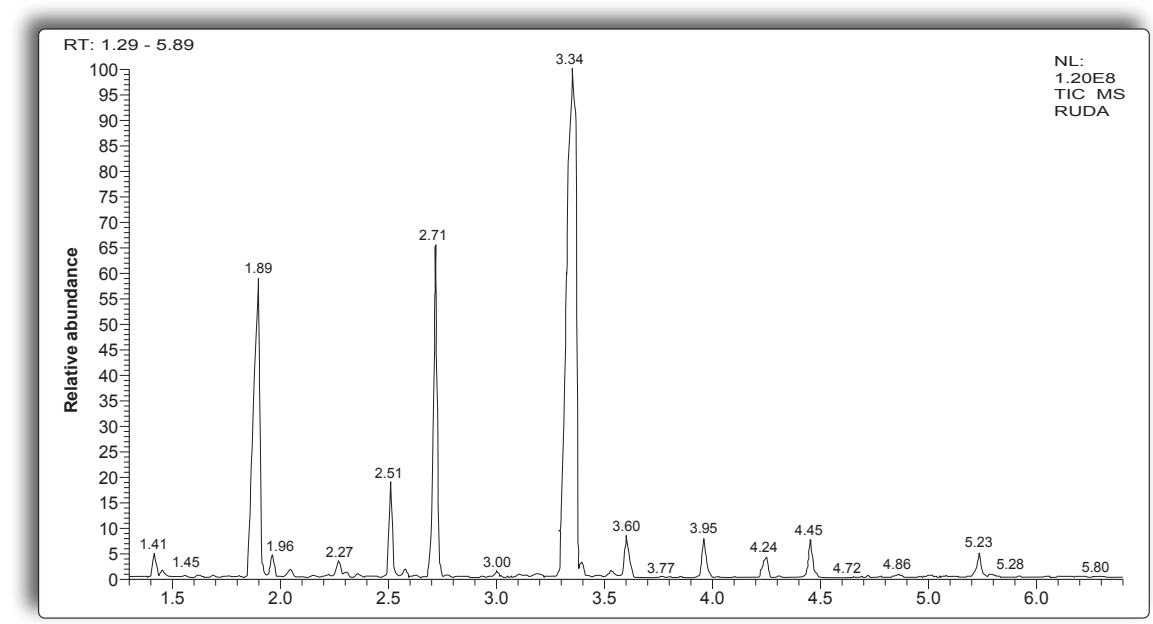

Figura 1. Cromatografía de Gas del aceite esencial de Ruta graveolens L. 


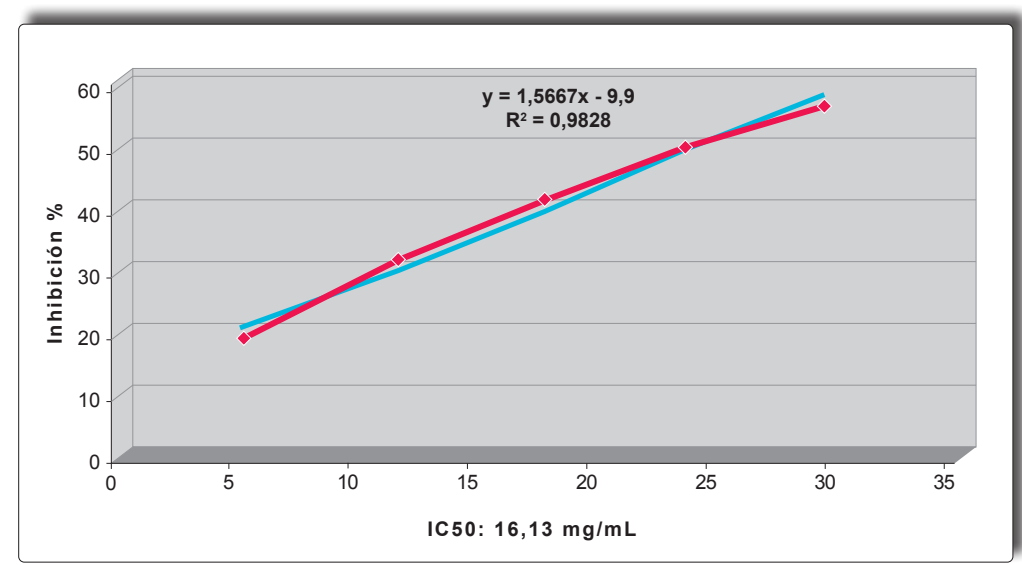

Figura 2. Porcentaje de captación de DPPH del aceite esencial de Ruta graveolens L.

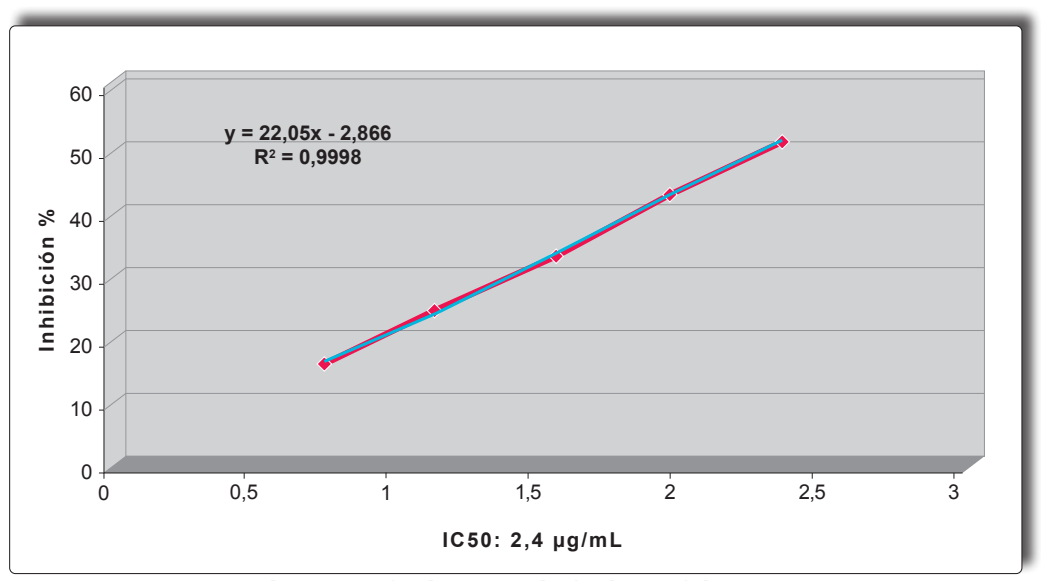

Figura 3. Porcentaje de captación de DPPH de ácido ascórbico.

2,2-difenilpicrilhidrazil (DPPH), se debería a la no presencia de compuestos terpénicos, sesquiterpénicos y aromáticos, ya que presenta compuestos mayoritariamente alifáticos y de la serie cetónica C-8 a C-13, además de otros hidrocarburos de cadena abierta y cerrada. Los resultados obtenidos demuestran que su actividad antioxidante es relativamente baja, presentando un IC 50 de 16,13 mg/mL frente al ácido ascórbico de 2,4 $\mu \mathrm{g} / \mathrm{mL}$.

Un estudio con extracto etanólico de Abuta grandifolia (Mart.), poseedor de alcaloides de tipo amonio cuaternario, mostró actividad citotóxica sobre embriones de Tetrapygus niger, erizo de mar negro ${ }^{(9)}$. El estudio del ciclo reproductivo de esta especie ha permitido realizar trabajos de actividad citotóxica en sustancias químicas ${ }^{(10)}$. La determinación de la actividad citotóxica de Ruta graveolens L. en el presente estudio demostró que a concentraciones de 50 y $75 \mu \mathrm{L}$, del aceite esencial, manifiesta citotoxicidad en el desarrollo embrionario in vitro de Tetrapygus niger.

\section{CONCLUSIONES}

En el aceite esencial de hojas frescas de Ruta graveolens L., se identificaron los siguientes componentes químicos: 2-octanona, 2-nonanona, nonanal, ciclohexen-5,6-dietenil1-metil, 2-decanona, metil nonyl éter, 2-undecanona, ciclotetradecano, 2-dodecanonay 2-tridecanona.

La evaluación de la actividad antioxidante in vitro del aceite esencial manifestó un $\mathrm{IC}_{50}$ de $16,13 \mathrm{mg} / \mathrm{mL}$, siendo un valor elevado comparado con el ácido ascórbico de $2,4 \mu \mathrm{g} / \mathrm{mL}$, por lo que la captación es relativamente baja.

El aceite esencial de Ruta graveolens L., mostró citotoxicidad, a concentraciones de 50 y $75 \mu \mathrm{L}$, en el desarrollo embrionario de Tetrapygus niger, erizo de mar negro.

\section{REFERENCIAS BIBLIOGRÁFICAS}

1. Morton JF. Atlas of medicinal plants of Middle America, Bahamas to Yucatan. Vol I. Charles C. Thomas Publisher. Illinois, 1981. p. 378-9.

2. Domínguez X. Métodos de investigación fotoquímica. 3ra Edición. Editorial Limusa. México DF, 1985.

3. Lock de Ugaz O. Investigación fotoquímica. Métodos en el estudio de productos naturales. Fondo Editorial Pontificia Universidad Católica del Perú. Lima, 1988.

4. Meccia G, Rojas BU. Estudio del aceite esencial de Ruta graveolens L. que crece en el estado Mérida - Venezuela, Instituto de investigaciones de la Facultad de Farmacia y Bioanálisis (IIFFB). Universidad de los Andes. Sector Campo de Oro, Mérida. 2009.

5. De Feo V, De Simone F, Senatore F. Potential Allelochemicals from the essential oil of Ruta graveolens. Phytochemistry, 2002; (61) 5: 573-8.

6. Dob T, Dahmane D. Volatile constituens of the essential oil of Ruta chalepensis L. Subsp. Angustifolia (Pers.) P. Cout. J Essent Oil Res, 2008; 20: 306-09.

7. Callapiña M, Jaimes N. Actividad mutagénica de los extractos acuosos de Ruta graveolens L. "Ruda" y Spartium junceum L. "Retama". Trabajo de aptitud Profesional para optar el Título Profesional de Químico Farmacéutico]. Facultad de Farmacia y Bioquímica UNMSM. Lima, 1995.

8. Amiel D. Aislamiento e identificación de alcaloides mutagénicos de las hojas de Ruta graveolens L. por métodos espectroscópicos UV-IR. [Tesis para optar al Título Profesional de Químico Farmacéutico]. Facultad de Farmacia y Bioquímica UNMSM. Lima, 1999.

9. Rojas Y, Soto R. Actividad citotóxica y efecto antitumoral de un extracto etanólico libre de alcaloides solubles en éter etílico del tallo de Abuta grandifolia (Mart.) Sandwith "Abuta" en líneas celulares. [Tesis para optar al Título Profesional de Químico Farmacéutico]. Facultad de Farmacia y Bioquímica UNMSM. Lima, 2003.

10. Zamora S, Stotz W. Ciclo reproductivo de Tetrapygus niger en dos localidades de la IV Región. Universidad de Chile. Coquimbo, 1993.

Manuscrito recibido el: 10/05/2011

Aceptado para su publicación el: 23/05/2011

\section{Correspondencia:}

Nombre: Américo Jorge Castro Luna

Dirección: Jr. Puno 1002 - Lima 1 - Perú.

e-mail: caslasha3@hotmail.com 